\title{
Relações entre Subdesenvolvimiento e Desenvolvimiento ${ }^{1}$
}

\author{
André Gunder Frank ${ }^{2}$
}

1 de julho, 1963

Proposito para um curso de pós-graduaça para o segundo semestre de 1963 no Departamento de Ciências Humanas da UnB

Este curso é uma tentativa de comprender as raizes, os mecanismos e a manutenção do subdesenvolvimiento através de cinco siglos do desenvolvimiento capitalista. A teoria e política de desenvolvimiento será basado no estúdio da realidade do subdesenvolvimento.

Nesto, o curso se distingua dos cursos ortodoxos de desenvolvimiento que presuponen que o subdesenvolvimento e apenas um estado preexistente ao, ou uma falte de, desenvolvimiento e que então encocan o problema do desenvolvimiento como uno de apenas seguir a estrada dos países desenvolvidos para cima. Pois, o curso é uma tentativa de enfocar o desenvolvimiento e sobre todo o subdesenvolvimiento não oleando da metrópole desenvolvida mas do punto de vista do mundo subdesenvolvido, e de America Latina e do Brasil em especial.

Relativamente novo como é este enfoco, o suas bases teoréticas ainda não são fracas se destacan com este enfoco apenas uma meia dúzia de autores como Baran, Myrdal, Lacoste, Furtado, Perroux e a revista Tiers Monde. Apenas umo delos e dum país subdesenvolvido. Os Marxistas tambem se distingan por a sua ausencia. Assim, vamos a ter nos mesmos que olear a realidad do desenvolvimiento/subdesenvolvimiento e tambem nos mesmos que construir a nossa propria teoria. O curso toma a sua tarefa tal revisão da realidad historica e presente, tal tarefa de descobrir umos principios de explicação desta realidad, e a confrontação com estos das teorias ortodoxas do desenvolvimiento que ainda gozam de acceptação comunmente demais. Assim, o proposito do curso e menos umo de dar respostas a perguntas velhas que o de levantar enfocos e perguntas novas para o futuro estudio e pesquisa.

\footnotetext{
${ }^{1}$ Agradecemos o esforço do prof. Juan Cristóbal Cárdenas Castro em recuperar o documento e cedê-lo para publicação na revista, sem tal trabalho inestimável não seria possível sua divulgação. A proposta de curso é caracterizada pela mistura de termos em castelhano e em português, com vários erros de digitação em máquina de escrever e alguns termos ilegíveis, procuramos manter a maior fidelidade possível ao documento original não realizando qualquer correção/falsificação do mesmo.

${ }^{2}$ Curta Biografia.
} 
Sera conveniente para a organização deste curso duas aulas por semana, uma de 2 horas de conferencia mina no principio da semana e outra no fim da semana para discussão da mina exposição e dos textos sobre o tema da semana. Sera conveniente que començando com a terceira semana os demais participantes tambem toma parte da responsibilidad da guia da aula de discussão, umo ou dois dellos preparando cada semana ilustraçoes do tema geral da semana para um pais o caso determinado, ou mesmo dando um enfoco que choca com a exposição mina.

Dada a poca disponibilidad de materiais e ainda mais de exemplares na nossa biblioteka, sera necessário para um curso no nível departamental uma certa disciplina dos participantes de usar os materiais em bom tempo e de fazeros disponibles a otros. Também será necessário uma mayor capacidad de, e possibilidad de confiança na, mecanografia que no primer semestre. Sera conveniente ter auxilio de pesquisa e sectretarial para mi no levantamento de materiais e dados para as conferencias, na pesquisa e o escrever relacionado ao tema do curso, e na preparação posterior em forma escrita das conferencias do curso. Sera conveniente uma sala para a centralisação dos materiais bibliográficos e se possível dos demais atividades do curso. Dada a importância do problema do subdesenvolvimento como produto simultâneo do desenvolvimiento e da dada ainda gran falta de estudios sobre esta relação, e consequentemente sobre as necesidades duma politica de superar ou liberar-se do desenvolvimiento/subdesenvolvimento, sera conveniente tambem que este tema e os diversos problemas a ser apontados no curso sejan incluidos tambem no programa de pesquisa do Centro de Pesquisa de Desenvolvimiento da UNB.

Os temas do curso e alguns materiais de leitura preliminares propostas e encontram a seguir. 
RELAÇÕES ENTRE O SUBDESENVOLVIMIENTO E DESENVOLVIMIENTO

A REALIADADE

I O SUBDESENVOLVIMIENTO NÃO E APENAS A FALTA DE DESENVOLVIMIENTO

1. A REALIDAD DO SUBDESENVOLVIMIENTO SUBODRINADO E

Lacoste (Os Paises Subdesenvolvidos)

Os Caracteres constitutivos do subdes.

Tipos de paises subdesenvolvidos

Myrdal (Teoria Economica e Regioes Subdes)

Caracteristica geral inexplicada da reald.

Prebish El Desarrollo de A. L. e sus problemas princps.

Arnault (Hist do Colorialismo) Ensayo de Balance

2. REALIDAD E UMA TEORIA DO SUBDESENVOLVIMIENTO

Lacoste - As causas originais do subdesenvolv.

Baran - As Raizes do subdes. (Ec. Pol. Do Des. Ec.)

Furtado - Elementos duma teoria do subdes. (Des.\&Subs.)

Prebish - El desarrollo de A.L. e otras obras

Perroux - La Coexistencia Pacifica

Tiers Monde - Sousdevelopment el development

Simoes - O Regional no subdes. Ec.

Singer - Distrib. Of Gains Between invest \&borrow. Counts.

\section{O MITO DO DESENVOLVIMENTO AUTONOMO}

Rostow As Etapas di Desenvolvimiento Economico

\author{
1. O MITO DO DESENVOLVIMIENTISMO-O BURGUES E O MARXISTA \\ Rostow - As Etapas di Des. Ec. \\ Lewis - A Teori do Desenvol. Economico \\ Higgins - Economic Development \\ Academia de Ciencias de URSS- Manual de Ec. Politica \\ Sweezy - Teoria do Desenvolv. Capitalista \\ ONU - Medidas para fomentar el desarrollo economico
}

Mead - A Neoclassical Theory of Economic Growth

Hagen - Social Factors in Economic Development (?) 


\section{RAIZES DO SUBDESENVOLVIMIENTO}

3. DESTRUCÇÃO DE SOCIEDADES VIABLES (A REALIDADE DE) E

See - As Origens do Capitalismo Moderno

Pirenne - Hist. Ec. E social da Edade Media

Huberman - H'st da Riqueza do Homem

Las Casas - Historia de las Indias

Mariategui - 7Ensayos sobre a realidad Peruana

Dutt - India Today

Marx - British Rule in India

Lattimore-Industrial Impact on China

Woddis - Africa: As raizes da revolta

A REALIDAD DO

4./MERCANTILISMO E A CONSTITUIÇÃO DE SOCIEDADES DE= PENDENTES

Bagú-Econ.da soc. colonial-Ensayo da hist..comp.A.L.

Williams-Capitalism and Slavery

Jullien-Les Decouvers maritime e le commerce du Levant

Arnault- Hist do Colonialismo

Furtado-Formaçao Economic deo Brasil

C. Prado Jr. - Hist. Ec. Do Bras. Form. do Brasil Contemp.

Simonsen- Historia Ec. do Brasil

Waibel-Capitulos de Geograf. Tropical e do Brasil

Frank-Agricult. Braz: Capitalismo e o Mito do Feudalismo

\section{O MYTO DO SUBDESENVOLVIMIENTO PRE=EXISTENTE}

- Brasil, India, Japon

\section{Hagen}

Lewis - A Teoria do Desenvolv. Economico
E

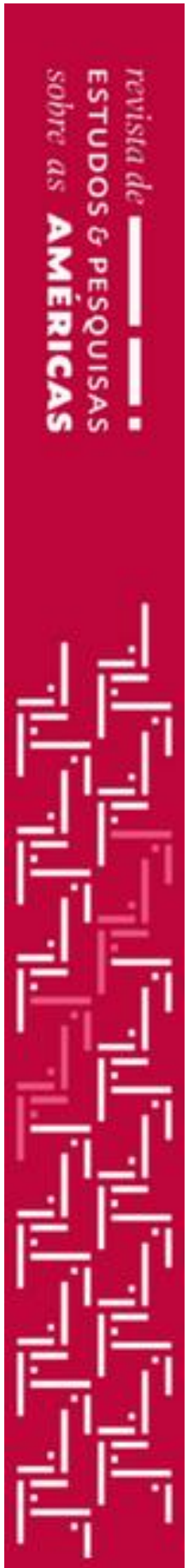

Revista de Estudos e Pesquisas sobre as Américas V.12 N.2 2018 ISSN: 1984-1639 
III;MECANISMOS CLASSICOS DO SUBDESENVOLVIMIENTO

\section{5; AREALIDAD DO CAPITALISMO MONOPOLISTA}

Dobb- Estudios no Desenvolvimiento Capitalista

Woddis-Africa: As Raizes da Revolta

Strachey- El Capitalismo Contemporane

\section{A REALIDAD DO COLONIALISMO E IMPERIALSIMO CLASSICO}

Deer- History of Sugar

Manchester- British Preeminence $n$ Brazil. Rise \& Decline

Phelps- Migration of Industry to South merica

Rippy-British Investiment in Latnn America, 1822-1949

Lenin- Imperialismo, Estado Superior do Capitalismo

PanikkerL'Asie el la domination occidentale

Brunschwig- La colonisacion francaise

Myrdal- [Ilegível] Ec E Reg. Subdes)-sobre desigulaidades

Bukharin - o imperialismo e a economia mundial

\section{A REALIDADE DE ETAPAS POLTICAS E SETORES ECONOMICOS} CRÍTICOS E DO DESENVOLVIMENTO RUSTADO

A.Pinto Sta. Cruz - Chile, Un caso de Des. Frustrado

Portugal e España - Porque são subdesenvolvidos?

Hirshcman - Estrategia do Desenvolvimento

Lenin- Estado e Revolução
Textosde Economia

Critica do marginalismo e do mercado livre

6 O MITO DA EGUALIZAÇÃO E DAS VANTAGENS COMPARATIVAS

Critiqa da teoria do egualisaçao no comercio internacional Critica [ilegível] das bases empiricas e da inconsistência teorética da $t$ teoria de costos/vantagens comparativas [ilegível] no tempo de Ricardo e depois

Nurkse-Proble s of Capital Formation 
Perroux-A empresa motora... e a região motora 8. DESEQUILIBRIO REGIONAL E SETEOAL (AREA DAD DE) Simoes-O Regional no Subdesenvolv. Econ.

Perroux-A empresa motora... e a região motora

\section{MANTENUAÇÃO DO SUBDESENVOLVIMIENTO}

9. A REALIDAD DA ECONOMIA DO MONOEXPORT E SERVICIOS CEPAL- La Economia de Venezuela en el ultimo decênio Hoselitz-Economic Growth in Latin America CEPAL - Estudios Varios

Di Tella-Ec. Y Estruct. Occupacional en un pais subdes.

10. A REALIDAD DO SUBDESENVOLVIMIENTO E INFLAÇÃO

A verdadera estrutura do desenvolvimiento, a falsa Distinção entre monetaristas e "estruturalistas" e demonstração da similitud fundamental das bases teoréticas e implicaçoes po it cas entre os dois

11. A REALIDAD DO COMMERCIO? “AJUDA' E NEOCOLONIALISMO

A. Frondizi - A Luta Antiimperialista

12. REALIDAD DOS EFFEITOS DA DEMONSTRAÇÃO DOSLIBERALISMOSE ECONOMICO; POLITICO; E SOCIAL
8. O MITO DA SOCIEDAD DUAL

Schatz-Um modelo de economia dualista de pais subdese. 9. O MITO DAS CONDIÇOES DO PROCESSO ECONOMICO Clark- Les Condicions du Progres Economique
10. O MITO DAS DIFERENCIA ENTRE AS RESERVAS [ILEGÍVEL] MONETRARI TAS E ESTRUTURALISTAS

Grunewald, Felix, Pinto, etc. vc. A politica do IMF
E

11. O MITO DA LIBERAÇÃO COLONIAL e DO CAPITAL COMO FALTA PRINCIPAL PA [ILEGÍVEL] O DESENVOLVIMIENTO

12. O MITO DO LIBERALISMO NO “MUNDO LIBRE”
Germani- Como fomentar las classes medias en A.L. 
